\title{
Pattern of a Flush-Mounted Microwave Antenna
}

\author{
James R. Wait
}

\begin{abstract}
The numerical results for the far zone radiation from an axial slot on a circular cylinder of perfect conductivity and infinite length are discussed. It is shown that the results for large diameter cylinders can be expressed in a universal form that is suitable for pattern calculations for arrays of slots on a gently curved surface. The work is compared with a related diffraction problem considered by Fock.
\end{abstract}

\section{Introduction}

The radiation from flush-mounted microwave antennas has aroused considerable interest in recent years $[1-6]{ }^{1}$ From a theoretical standpoint the problem would seem to be simple enough; the surface on which the antenna is mounted is considered to be adequately represented by a sphere or cylinder whose radius of curvature is matched to that of the local curvature of the actual surface. Unfortunately, the formal solution of the problem, in terms of the classical harmonic series involving integral or halfintegral order Bessel functions, always converges very poorly when the radius of curvature is large compared to the wavelength. There are two wellknown alternative representations $[6,7]$, however, which can be used to advantage in certain cases. In the illuminated region of the antenna, geometrical optics is most satisfactory, while deep in the shadow the rigorous harmonic series can be converted into the rapidly converging residue series.

The calculation of the radiation field of a flushmounted antenna in the tangent plane (the classical light-shadow boundary) is not readily treated by either geometrical opties or the residue series. In the former case the field is indeterminant, and in the latter case the convergence is extremely poor and would actually diverge in the illuminated region. Despite the fact that the harmonic series is cumbersome, it is valid in this transition zone between the illuminated and shadow regions of space. Therefore, it is desirable to attempt to adapt the harmonicseries representation to surfaces of large radius of curvature. This is the purpose of the present paper.

\section{Theoretical Basis}

A thin axial slot, cut on a circular cylinder of infinite length and perfect conductivity is considered because it gives rise to a plane-polarized radiation field. The cylinder is taken to be of radius $a$ and coaxial with a cylindrical coordinate system $(\rho, \phi, z)$. The slot that extends from $z_{1}$ to $z_{2}$ at $\phi=0$, has a voltage distribution $V(z)$ throughout its length. The radiation pattern is best expressed in terms of spherical coordinates $(r, \phi, \theta)$ where $\theta=0$

Figures in brackets indicate the ${ }^{1}$ literature references at the end of this paper corresponds to the axis of the cylinder. It has been shown [9] that the electric field, which has only a $\phi$ component, is given by

$$
E_{\phi}=\frac{e^{-i k r}}{r} S(\theta) M(x, \phi),
$$

where

$$
\begin{aligned}
& S(\theta)=\frac{k \sin \theta}{2 \pi} \int_{z_{1}}^{z_{2}} V(z) e^{i k z \cos \theta} d z, \\
& M(x, \phi)=\frac{1}{\pi x} \sum_{m=0}^{\infty} \frac{\epsilon_{m} e^{i m \pi / 2} \cos m \phi}{H_{m}^{(2) '}(x)},
\end{aligned}
$$

$x=k a \sin \theta, k=2 \pi /$ free space wavelength, $\epsilon_{0}=1, \epsilon_{m}=$ $2(m \neq 0)$, and $H_{m}^{(2) \prime}(x)$ is the derivative of the Hankel function appropriate for a time factor $\exp (i \omega t)$. Equation (1) is valid for $k r \sin \theta \gg 1$.

The function $S(\theta)$ is the space factor of the slot, or an array of collinear slots if they were cut in an infinite plane conducting sheet. The function $M(x, \phi)$ is called the "cylinder space factor" as it fully describes the effect of the finite diameter of the cylinder on the radiation pattern in both the $\theta$ and $\phi$ directions. For purposes of computation, it is written

$$
M(x, \phi)=|M(x, \phi)| e^{i \alpha(x, \phi)} .
$$

The phase function $\alpha(x, \phi)$ is a rapidly varying function of $\phi$ for the larger $x$ values. For this reason, it is desirable to express $\alpha$ in the illuminated region $(0<|\phi|<\pi / 2)$ as a geometrical-optical term plus a correction factor $\Delta(x, \phi)$, as follows [9]

$$
\alpha(x, \phi)=\alpha(x, 0)-x(1-\cos \phi)+\Delta(x, \phi) .
$$

In the shadow region $(\pi / 2<|\phi|<\pi)$, it is desirable to express $\alpha$ as a physical-optical term plus a correction, which is also designated $\Delta(x, \phi)$, in the following manner.

$$
\alpha(x, \phi)=\alpha(x, \pi / 2)+(\pi / 2-\phi) x+\Delta(x, \phi) .
$$

The amplitude $|M(x, \phi)|$ and the phase correction $\Delta(x, \phi)$ of the cylinder space factor are shown plotted in figures 1 and 2 for $\phi$ from 0 to $180^{\circ}$ for $x=10,12$, 15,18 , and 21 . To prevent troublesome overlapping, the ordinates are shifted for each curve by a constant amount. 


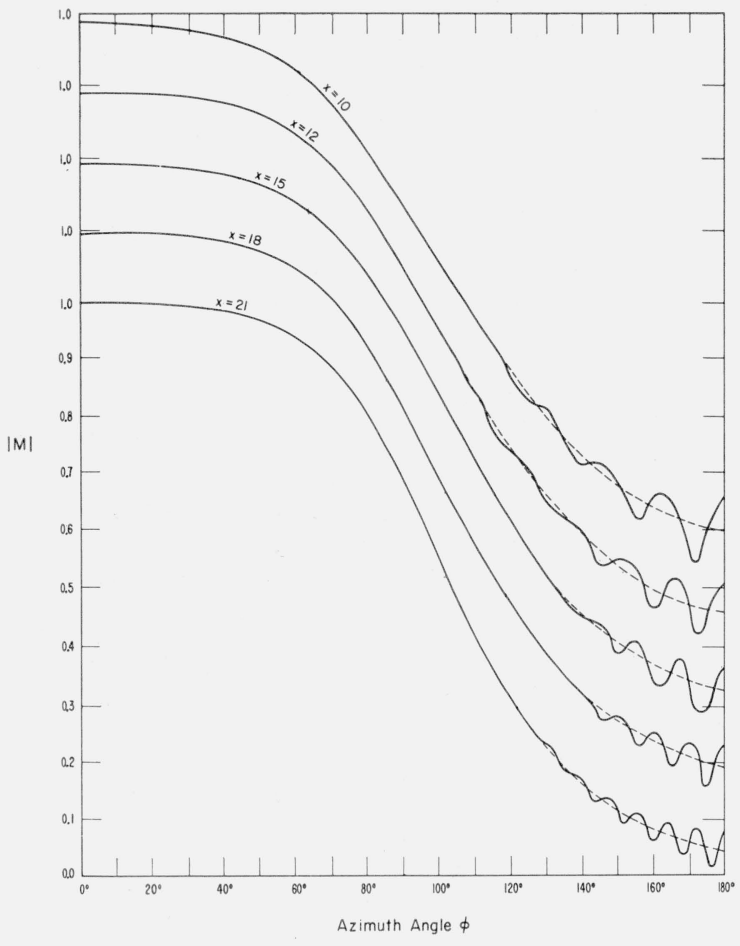

Figure 1. Amplitude of cylinder space factor for narrow axial slot.

N. B. vertical scale is shifted for each curve.

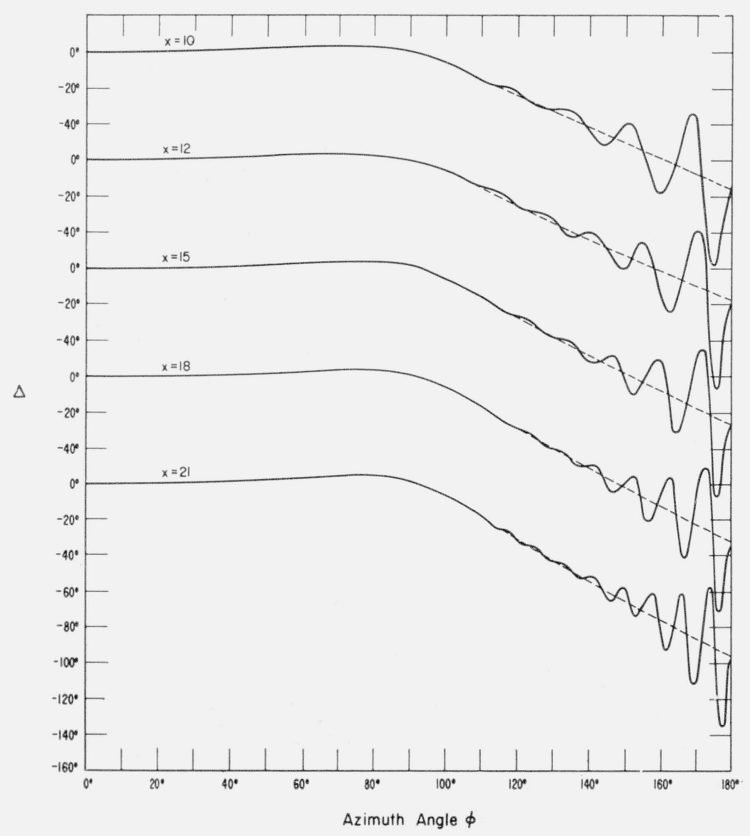

Figure 2. Phase of cylinder space factor for narrow axial slot. N. B. vertical scale is shifted for each curve.
For $|\phi|<90^{\circ}$ but not near $90^{\circ}$, the value of the space factor is quite well approximated by the geometrical-optics approximation. That is

and

$$
\Delta(x, \phi) \simeq 0
$$

$$
|M(x, \phi)| \simeq 1
$$

Deep in the shadow, that is $|\phi|>90^{\circ}$ but not near $90^{\circ}, M(x, \phi)$ has the form of a damped standing wave, and can be characterized by a function of the form

$$
A(\phi-\pi / 2) e^{-i x(\phi-\pi / 2)}+A(3 \pi / 2-\phi) e^{-i x\left(\frac{3 \pi}{2}-\phi\right)}
$$

where

$$
A(\beta) \cong \exp \left[-i \tau(\pi a / \lambda)^{1 / 3} \beta\right]
$$

with $\tau=0.808 \exp (-i \pi / 3)$. This exponential form for $A(\phi)$ is the first term of a rather complicated residue series [7]. It is only valid for $k a \gg 1$. The higher order terms (higher modes) are also parametric in $(\pi a / \lambda)^{1 / 3} \beta$, where $\beta$ is some angular distance. It is, therefore, logical to expect $M(x, \phi)$ to be parametric in $(x / 2)^{1 / 3}(\phi-\pi / 2)$ and $(x / 2)^{1 / 3}[(3 \pi / 2)-\phi]$. In fact, if the ripples are ignored, the function $M(x, \phi)$ should be a function only of $(x / 2)^{1 / 3}(\phi-\pi / 2)$. The dotted curves in figures 1 and 2 are believed to be the appropriate form of $M(x, \phi)$ when the standing-wave effect, due to the interfering traveling wave from the other side of the cylinder, is removed. These "smoothed" curves are then replotted as a function of $X$, where $X=(x / 2)^{1 / 3}(\phi-\pi / 2)$. The results are shown in figure $3 \mathrm{a}$ where both the values of amplitude $|\mathrm{M}(\mathrm{X})|$ and the phase correction $\Delta(X)$ for $x=10$, $12,15,18$ and 21 fall on the same set of curves for the range of $X$ indicated. The amplitude is also shown in figure $3 \mathrm{~b}$, being plotted on a log scale.

Having the space factor for the cylinder plotted in this universal form suggests that one may extrapolate the results to larger values of $x$. This concept, embodied in presenting the results in terms of a parameter $X$, is not unrelated to the notion of angular distance that has been successfully employed in the representation of field strengths of a transmitter at large ranges over a spherical earth [10]. In the latter instance, the angular distance is defined as the angle subtended at the center of the earth between the horizons of transmitting and receiving antennas. In the present situation, the angular distance is $\phi-\pi / 2$ since the source antenna is on the cylinder and the observer is at infinity.

While the present problem is concerned directly with the radiation of an axial slot on a circular cylinder, there is a direct application of the results to the reciprocal case where a plane wave is incident on the cylinder (the $E$ vector is to be perpendicular to the cylinder axis). Then it is possible to regard $M(x, \theta)$ as being proportional to the surface current excited on the cylinder. In other words, the axial slot can be regarded as a receiving antenna and the source is taken to be at infinity.

In view of the reciprocal nature of the problem, it is desirable to compare the present results with those 


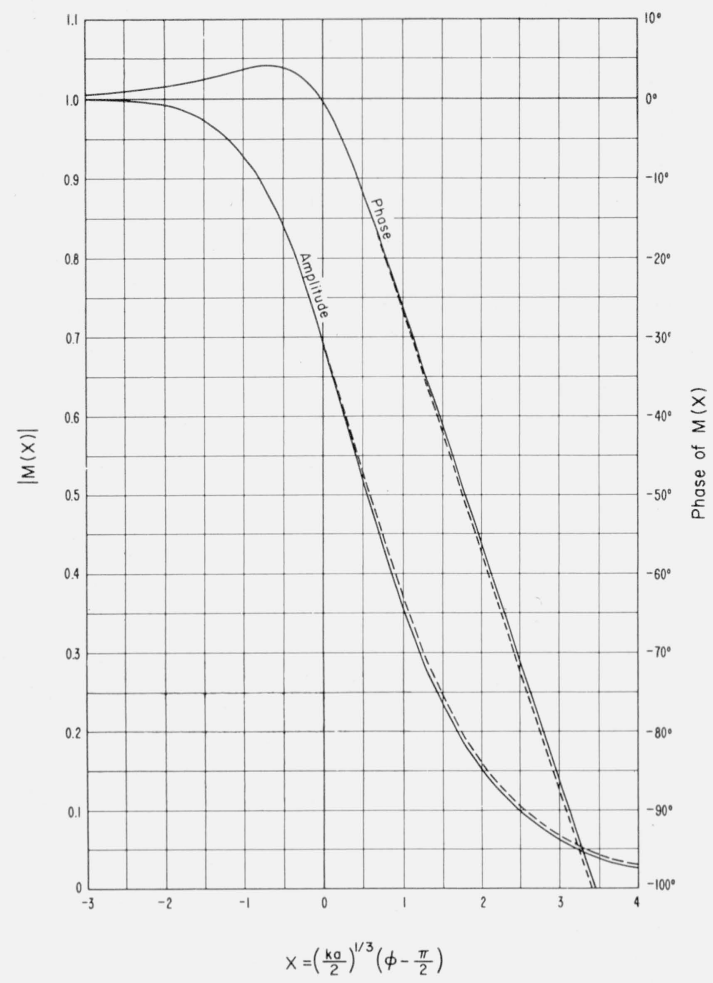

Figure 3a. Amplitude and phase of space factor in parametric form.

_ From eircular eylinder data; ...... from Fock.

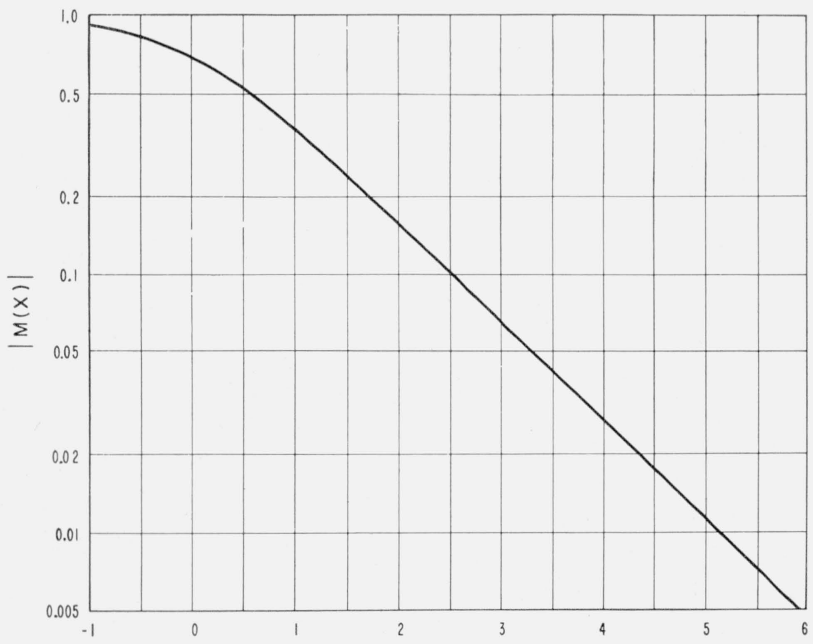

Figure 3b. Amplitude of space factor in parametric form.

From Circular - cylinder data. of Fock [11] for the currents excited on a curved surface by an incident plane wave. Fock first considers the diffraction of plane wave by a paraboloid and, in particular, focuses his attention on the tangential magnetic field in the penumbral region (near the boundary of light and shadow). Then, after postulating that the surface currents are only dependent on the local radius of curvature, he obtains a representation for the current distribution that is only dependent on the parameter $l / d$ where $l$ is the distance from the light-shadow boundary and $d$ is the width of the penumbral region. Fock then believes that his results are reasonably valid for any shaped bodies so long as the radius of curvature is always very large compared to the wavelength. As a crucial test of Fock's approximate formula, his results are plotted in figure 3a along with the cylinder computed data. The agreement is very good. There appears to be a slight discrepancy for larger positive $X$ values which is not entirely unexpected because some of Fock's restrictions are becoming violated. Nevertheless, considerable justification is given to the validity of the extrapolation of the cylinder curves to larger values of $x$.

\section{An Application}

An interesting application of the foregoing theory is the calculation of the pattern of an end-fire array, of slots on a gently curved surface. For example consider the array of $2 N+1$ parallel and axial slots on a cylindrical surface of radius $a$ indicated in figure 4. The angle $\Phi$ defines the direction of the observer

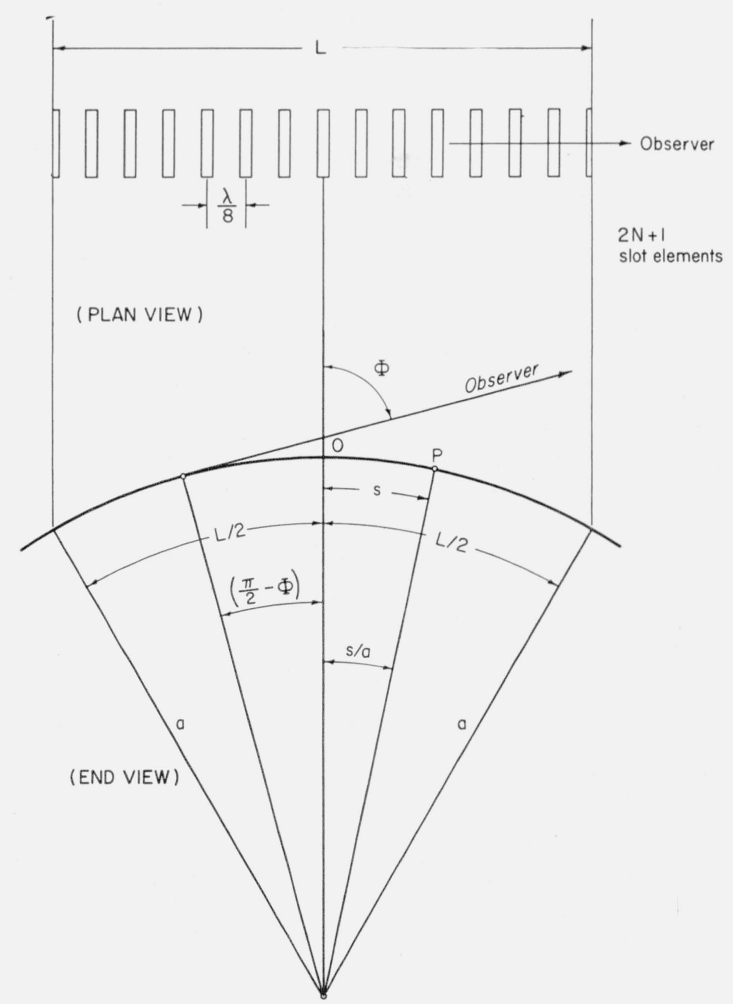

FIGURE 4. Schematic diagram of end-fire array of slots on a curved conducting surface. 
with respect to the normal at 0 , the center of the array. The array and the observer are taken to be in the principal plane (i. e., the plane of the paper). $s$ is the distance from 0 , measured along the cylinder surface, to $P$, the location of any one of the elements. The field due to the source at $P$ is then proportional to

$$
e^{i k y} K(s) A(s)
$$

where $K(s)$ is the relative strength of the element, $y$ is the projection of $s$ on the ray from 0 to the observer and $A(s)$ is the correction factor to geometrical optics. In terms of the function $M, A(s)$ is given by

$$
\begin{aligned}
& A(s)=M(X) \text { for } X \leqq 0, \\
& \simeq M(X) e^{-i \frac{X^{3}}{3}} \text { for } X \geqq 0,
\end{aligned}
$$

where

$$
X=-\left[\left(\frac{\pi}{2}-\Phi\right)+\frac{s}{a}\right]\left(\frac{k a}{2}\right)^{1 / 3}
$$

The factor $y$ is given by $y=c \sin [\Phi-(s / 2 a)]$ when $c$ is the chord length $O P$ and because

$$
c=\left[2 a^{2}\left(1-\cos \frac{s}{a}\right)\right]^{1 / 2} \cong s\left(1-\frac{s^{2}}{24 a^{2}}\right),
$$

it follows that

$$
y \cong s\left(1-\frac{s^{2}}{24 a^{2}}\right) \sin \left(\Phi-\frac{s}{2 a}\right)
$$

subject to $s<<a$.

Remembering that there are $2 N+1$ elements spaced at intervals $\Delta s$, it is seen that

$$
2 N \times \Delta s=L
$$

where $L$ is the length of the array. The source is now written

$$
K(s)=e^{-\Gamma s}
$$

where $\Gamma$ is the propagation constant of the exciting wave along the array. For the present purpose

$$
\Gamma=+i m k,
$$

where $m$ is a constant real number.

The total field of the array is now proportional to

$$
\sum_{n=-N}^{N} e^{i k y} e^{-i k m s} A(s)
$$

where $s=n \cdot \Delta s$ with $n=-N,-N+1, \ldots-1,0$, $1,2 \ldots N-1, N$. Introducing dimensionless parameters, defined by

$$
\Delta S=k \Delta s \text { and } S=n \Delta S
$$

it follows that the pattern $T(\Phi)$ of the array is given by

$$
T(\Phi)=\sum_{n=-N}^{N} e^{i[Y(S)-m S]} A(X)
$$

where

$$
Y(S)=S\left(1-\frac{S^{2}}{24(k a)^{2}}\right) \sin \left(\Phi-\frac{S}{2(k a)}\right)
$$

and

$$
X=-\left[\left(\frac{\pi}{2}-\Phi\right)+\frac{S}{k a}\right]\left[\frac{k a}{2}\right]^{1 / 3} .
$$

When $k a$ tends to infinity such that the surface is effectively flat, the pattern becomes

$$
T(\Phi)=T_{0}(\Phi)=\sum_{n=-N}^{N} e^{i[Y(S)-m S]}
$$

where $Y(S)=S \sin \Phi$ and since $\mathrm{S}=n \cdot \Delta S$,

$$
T_{0}(\Phi)=\frac{e^{-i N K}\left(1-e^{i(2 N+1) K}\right)}{1-e^{i K}}
$$

where $K=\Delta S[\sin \Phi-m]$.

According to the analysis of Hansen and Woodyard [12], the maximum gain in the end-fire direction (i. e., $\left.\Phi=90^{\circ}\right)$ is obtained when $m \cong(N+2) / N$. Then, taking the electrical spacing between the elements as $45^{\circ}$ or $\Delta S=\pi / 4$, the array has 65 elements, $N=32$ and the total length of the array is 8 wavelengths. The pattern $T_{0}(\Phi)$ in this case is only defined for the region $-90^{\circ}<\Phi<90^{\circ}$. The main lobe and the first side lobe are shown plotted in figure 5 normalized such that the maximum field is $0 \mathrm{db}$. Using the same values of $N$ and $\Delta S$, the corresponding pattern $T(\Phi)$ is plotted for $k a=200$. The main lobe is seen to be somewhat changed in

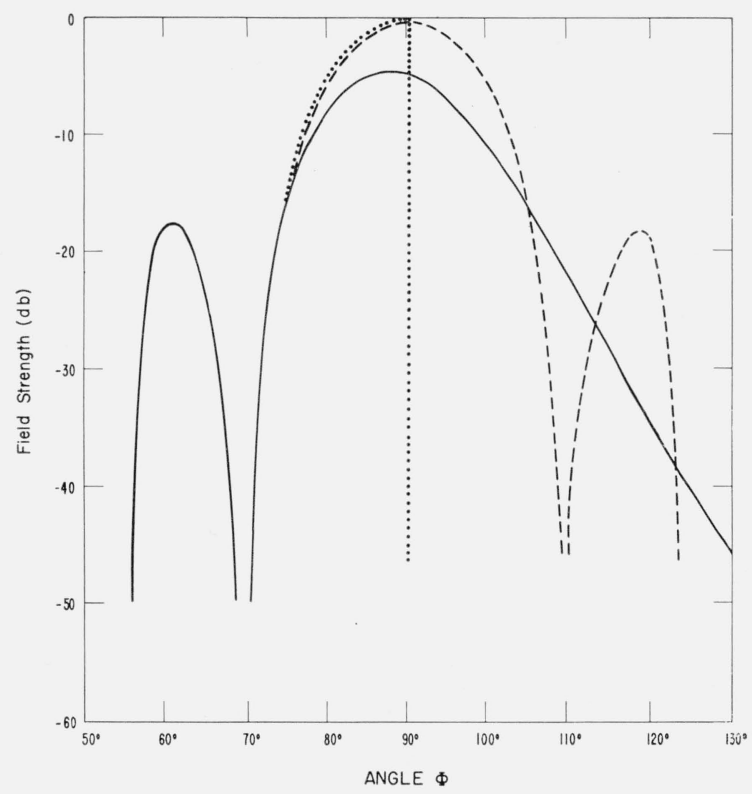

Figure 5. Pattern of end-fire array of slots on a curved surface. ber of elements $=65 . \quad k a=200 ;---k a=200, A(X)=1$; array length $=8 \lambda$; num- 
form, particularly in the directions near $\Phi=90^{\circ}$ which is the tangent plane of the center of the array. The decibel level of the field in the diffraction zone $\left(\Phi>90^{\circ}\right)$ is seen to decrease linearly with angle. There are no lobes formed in this region. It is of interest to know how the curvature of the array itself would modify the pattern. Therefore, for sake of comparison, the pattern $T(\Phi)$ of the endfire array of isolated elements on a circular arc $(k a=200)$ is also plotted in figure 5. The working formula here is identical to equation (8a) with $A(X)$ being replaced by 1 because diffraction effects are no longer present. The pattern is almost identical to that for an end-fire array where elements are on a straight line. Lobes, in this case, are formed on both sides of the main beam although they are not quite symmetrical about it.

The pattern $T(\Phi)$ is also computed for $k a=100$, and is shown plotted in figure 6 along with the curve for $k a=\infty$ and the curved array of isolated elements.

It would seem that an end-fire array of slots on a curved surface has some rather interesting properties. The main effects of the non-flatness of the surface is to tilt the maximum of the main beam up slightly, and to extend the total width of the main beam into the shadow region. There is no evidence of lobes on this side of the beam, although at large angles ( $\Phi$ approaching $180^{\circ}$ ), there is the possibility that the back lobe from the end-fire array could creep around the surface in the other direction and form lobes. This effect trom a practical standpoint, however, is completely insignificant for $k a>100$.

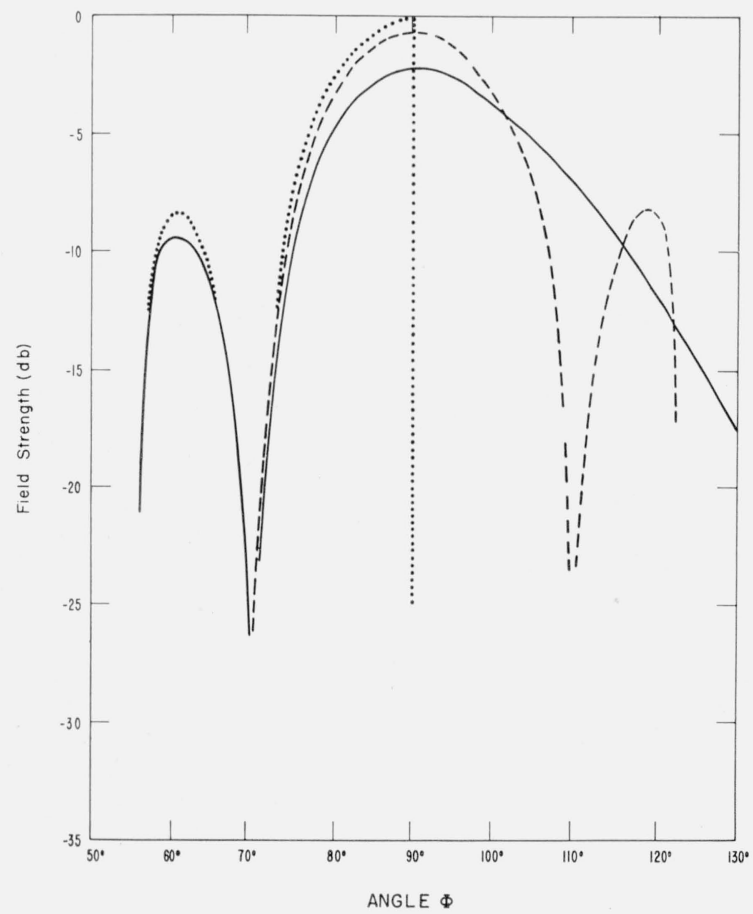

Figure 6. Pattern of end-fire array of slots on a curved surface. $\ldots \ldots k a=\infty ;-k a=100 ;--k a=100, A(X)=1$; array length $=8 \lambda$; num-

\section{Concluding Remarks}

It is seen that the radiation pattern of a flushmounted antenna is influenced, to a considerable extent, by the radius of curvature of the surface on which it is mounted. In most cases, the main beam is tilted upward away from the surface, although the total width of the beam can be broadened into the shadow. Similar phenomena had been observed in the calculated patterns of slots on a conducting halfplane [13]. It would appear that any attempt to reduce the side lobes of an end-fire array by using a diffracting edge or surface will lead to a broadening of the main beam. It is also quite apparent that the resultant pattern of a flush mounted antenna is not simply obtained by multiplying the free space pattern by the pattern of a single slot on the curved surface. Such a process would produce a pattern with lobes in the diffraction or shadow region which do not exist in reality for a nonclosed surface. Furthermore, this "multiplication" technique would not predict the broadening of the beam in the tangent plane.

The author thanks William Briggs for his assistance in the calculation of the patterns in figures 5 and 6 .

\section{References}

[1] G. Sinclair, The patterns of slotted cylinder antennas, Proc. I. R. E., 36, 1487 (1948).

[2] C. H. Papas, Radiation from a transverse slot in an infinite eylinder, J. Math. and Phys. 28, 227 (1950).

[3] C. Silver and W. K. Saunders, Radiation from a transverse slot in a circular cylinder, J. Appl. Phys. 21, 749 (1950).

[4] S. Sensiper, W. G. Sterns, and T. T. Taylor, A further study of the patterns of single slots on circular conducting cylinders, paper presented at Spring International Scientific Radio Union (U. R. S. I.) Meeting (April 24, 1951).

[5] J. R. Wait and S. Kahana, Calculated patterns of circumferential slots on a circular conducting cylinder, Can. J. Technol. 33, 77 (1955).

[6] L. L. Baillin, The radiation field produced by a slot in large circular cylinder, Trans. P. G. A. P. (Institute of Radio Engineers) 3, 128 (1955).

[7] G. N. Watson, The diffraction of radio waves by the earth, Proc. Roy. Soc. 95, 546 (1919).

[8] H. Bremmer, Terrestrial radio waves, theory of propagation, chapter 3 (Elsevier Publishing Co., Amsterdam, 1949)

[9] J. R. Wait, Radiation characteristics of axial slots on a conducting cylinder, Wireless Engr. 32, 316 (1955).

[10] K. A. Norton, P. L. Rice, and L. E. Vogler, The use of angular distance in estimating transmission loss, Proc. I. R. E. 43, 1488 (1955).

[11] V. Fock, The field of a plane wave near the surface of a conducting body, J. Phys. (U. S. S. R.) 10, 399 (1946).

[12] W. W. Hansen and J. R. Woodyard, A new principle in directional antenna design, Proc. I. R. E. 26, 333 (1938).

[13] J. R. Wait and R. E. Walpole, Calculated radiation characteristics of slots cut in metal sheets, Can. J. Technol. 33, 211 (1955).

Boulder, Colo., March 28, 1957. 